\title{
HYBRID ADDITIVE MANUFACTURING - REQUIREMENTS ENGINEERING FRAMEWORK FOR PROCESS CHAIN CONSIDERATIONS
}

\author{
Schneberger, Jan-Henrik (1); Häfele, Tobias (2); Kaspar, Jerome (1); Vielhaber, Michael (1) \\ 1: Saarland University, Institute of Engineering Design, Germany; 2: University of Applied Sciences \\ Saarland, School of Engineering, Germany
}

\begin{abstract}
Additive Manufacturing (AM) provides significant opportunities for design and functional integration of parts and assemblies. Compared to conventional processes, the AM principle increases design freedom notably. Additionally, numerous processible materials and hybrid processes enable the implementation in different industries, spanning from aerospace over automobile until medical applications.

However, there are still handicaps to be addressed, arising from the large diversity of AM principles, post-processing and quality assurance issues, partly insufficient user knowledge, and organizational aspects. Coherently, lacking requirements specification hinders a successful consideration of AM in the early stages of development, and its later implementation.

To promote knowledge build-up, this contribution presents a requirements specification framework, which supports developers in determining demands throughout the development process, including those resulting from post-processing and testing operations. By incorporating thorough analyses of general organizational and resort overarching limitations, this contribution promotes a successful implementation of suitable AM strategies.
\end{abstract}

Keywords: Additive Manufacturing, Requirements, Design process, Process chain design

Contact:

Schneberger, Jan-Henrik

Saarland University

Institute of Engineering Design

Germany

schneberger@1kt.uni-saarland.de

Cite this article: Schneberger, J.-H., Häfele, T., Kaspar, J., Vielhaber, M. (2019) 'Hybrid Additive Manufacturing Requirements Engineering Framework for Process Chain Considerations', in Proceedings of the 22nd International Conference on Engineering Design (ICED19), Delft, The Netherlands, 5-8 August 2019. DOI:10.1017/dsi.2019.372 


\section{INTRODUCTION}

Additive Manufacturing (AM) gains significantly increasing importance in the scientific as well as industrial community. Due to its large diversity regarding process principles and its extensive material repertoire, AM technologies offer the opportunity to select the best-fitting combination of the aforementioned elements for any given task in engineering design and manufacture (Herderick and Patterson, 2016). AM is already widely adopted as a rapid prototyping technology and is gradually gaining more impact on rapid manufacturing of net-shape or near-net-shape components. Following the observations of Milewski (2017), this trend predominantly occurs in the sensitive aviation and automotive industries, as well as medical applications. The indisputable advantages, which can be uncovered by the use of $\mathrm{AM}$ in prototyping and production, are emphasized throughout the scientific literature (e.g., Gibson (2015) and Milewski (2017)), but also in books and magazines for casual application and consumers (e.g., Deloitte (2017)). Coming along with these multi-facetted enablers, various new challenges arise from different implications. First, the unconventional principles and opportunities of AM processes impede a stringent one-by-one adoption in conventionally characterized process chains, as indicated by Loy and Tatham (2016) and Gebhardt (2016). Second, challenges and inconveniences take roots in the industrial and societal effects of both, development and manufacture of products (Loy and Tatham, 2016). A further aspect is the need for sufficient process stability and qualification of process and work piece right before the admission to assembly and use-phase of the object (Sames et al., 2017). However due to the manifold possibilities offered by AM technology, customers and users are not necessarily familiar with their new freedoms. This results in uncertainty and refusal of an implementation of AM processes, which might actually leverage product value.

To address this issue appropriately, this contribution focuses on the need for specific requirements determination in the context of product design and process chain selection. Here, the authors initiate their investigation of a general framework for the determination and elicitation of relevant requirements in the earliest stages of product development where both - part and process requirements yield the most significant influence on the later process chain layout. The considerations do not only involve the primary AM process, but also hybridized AM process chains along with their respective impacts on postprocessing operations as well as (non-)destructive testing and qualification of parts and assemblies. In order to provide a manageable scope and illustrative examples, the contribution narrows the available spectrum of AM to laser-based processes. In particular, these include powder-bed based selective laser melting (SLM) as well as powder feedstock based laser metal deposition (LMD).

\section{STATE OF THE ART IN LITERATURE}

Aiming to present a thorough overview of the required methods and technologies considered in this contribution, the following section presents a recapitulation of the state of the art in literature. Starting with the aforementioned AM processes (SLM and LMD) the authors acquire and assess technological properties regarding chain integration capability and potential applicability. The subsequent paragraphs treat the fundamental requirements engineering approaches in engineering and those considering AM in particular.

\subsection{Laser-based additive manufacturing technologies}

According to the ASTM standards (ISO/ASTM, 2015), AM processes are divided into seven categories, which classify the processes, for example, according to the type of feedstock material and tool principle, as highlighted by Milewski (2017). The widespread spectrum allows for the determination of suitable processes (e.g., by assessing specific degrees of feature detail or processing speed) and materials (e.g., based on future application and economic boundary conditions) in both rapid prototyping and rapid manufacturing. Focusing on laser-based processes using powder as feedstock material, the predominant principles are powder bed fusion and directed energy deposition (ISO/ASTM, 2015). Referring to Schmidt et al. (2017), the most prominent representatives have been adopted up to a significant degree of applicability. Due to their ability to produce durable and high quality (near-) net-shape components reliably, laser-based AM processes are of peculiar importance in the industrial environment, which is emphasized by $\mathrm{Gu}$ (2015) and Schmidt et al. (2017). However, there is still a lack of robustness, simplicity and process predictability, which restricts even more comprising employment. 


\subsubsection{Selective laser melting - principle and properties}

According to the ISO/ASTM (2015) standard, SLM is classified as a powder bed fusion process. Thus, parts are generated by a laser, which locally melts and sinters particles in a powder bed, leading to local agglomeration and solidification of the powder. The foundation for AM parts in the SLM process is laid by a rigid build platform that fulfils different functions at once. First, it acts as the mechanical anchor-plane for the part; second, it contributes to the process heat sink. Combined with support structures on the part, the build plate is necessary in order to lower or eliminate distortion and warping, as stressed by Gibson (2015). The platform is lowered gradually throughout the process, leading to the characteristic layer-wise build-up of the fabricated part. After lowering the platform, a recoater element (e.g., roller or scraper) spreads a new layer of powder, which is then melted by the laser beam. According to the respective material, the build chamber is flooded with process gas (commonly nitrogen or argon) which acts as a fundamental process enabler. On the one hand, it provides the necessary, low oxygen concentration required for best-possible melt pool quality and stability. Due to a permanent gas exchange and filtration process in a nearly laminar stream above the powder bed, on the other hand, it also contributes to the evacuation of smoulder. SLM incorporates an enormous amount of parameters affecting the process and its outcome. Due to the major principle and the machines' mechanical constitution, macroscopic aspects, such as part position on the build plate, build orientation and height, lead to considerable impacts on build time and quality (Adam, 2015; Byun and Lee, 2006). Additional parameters (e.g., layer height, major scanning strategy, and laser focal diameter) contribute to the above issues at least equally. Going into detail even further, hatching distance and orientation, as well as adaptive laser power output yield strong influence on parts' thermal and mechanical conditions, but also on the material's microstructure ( $\mathrm{Gu}, 2015$; Sames et al., 2016). All these aspects highlight the complexity and diversity of the process and need to be taken into consideration when applying AM processes in a production environment. This additionally is topped by a manifold application of tailored material research conducted on the development of new materials (including ceramic alloys and metallic glasses), enhanced parameter settings and general parameter prediction (Gu, 2015; Mies et al., 2016).

\subsubsection{Laser metal deposition - principle and properties}

Laser metal deposition is classified in the category of Directed Energy Deposition (DED), which includes all processes, where focused thermal energy is used to melt a deposited material (ISO/ASTM, 2015). Depending on the process and machine configuration, the technology can be used for building small and large parts to repair and rebuild worn or damaged components and to generate wear and corrosion resistant coatings (Gu, 2015; Brandt, 2017). Instead of a pre-placed feedstock in SLM, the material is fed by a powder nozzle in LMD (coaxially or off axis), while a laser generates a melting pool on the substrate by means of a carrier gas (Lorenz et al., 2015). For the production of composites or functionally graded materials, different materials could be supplied simultaneously or sequentially (Mahamood, 2018). The materials include iron-based alloys, aluminum- and nickel-based alloys, titanium alloys and cobalt-based alloys, principally analogous to the SLM process. After solidification, single beads are formed, which could be placed next, or on top of each other to create layers or volumes. Thus, positioning of the substrate significantly influences the complexity of the components, while part dimensions are limited by the handling system. For processing of more complex geometries, substrates or parts can be positioned in a stationary position (three axis systems) or on a rotating stage (five-axis systems) described in Sames et al. (2016). Thereby, the properties of the component (e.g., microstructure) as well as productivity (e.g., buildup rate) are influenced by several parameters. According to Mahamood (2018), the key processing parameters are laser power, scanning velocity, powder flow rate, and gas flow rate. Coherently, Brandt (2017) and Graf et al. (2018) highlight the opportunity to apply DED for consistent and efficient powder delivery, but also stress the negative influences of the weld bead geometry produced. Since layer thickness is typically larger than in SLM, DED enables the production of near-net-shape geometries that need to be machined afterwards (Sames et al., 2016; Lorenz et al., 2015). The combination of build-up, repair and coating, as well as the respective post-processing steps, results in an enormous variety of applications for this additive process.

\subsection{Considering requirements engineering in additive manufacturing}

Considering the employment of $\mathrm{AM}$ in a development project implies the application of thorough requirements specification. AM is characterized by several differences compared to conventional 
processes. First, material and process are not separable at any stage. Material properties are created within the process, significantly influenced by individual process parameters. Second, the additive process principle induces noticeable design and layout shifts, corresponding to the layer-wise material deployment. Third, AM processes constitute a more or less revolutionary technology that has not gained full acceptance and control amongst manufacturers. Traditional routes, for example, foundry processes with downstream machining operations can be considered as established, also among workers occupied with the technology. In contrast, AM has not been introduced in companies up to the same level. Knowledge on the core process as well as downstream post-processing steps is often implicitly stored and limited to individuals. Largerscale education programs, however, are often exclusively provided to workers, who are directly confronted with the technology in a respective company. A standardised apprenticeship for the industrial sector has not yet been proposed. The aforementioned issues restrain adoption and consent among manufacturers. They shall be addressed by the RE framework presented in this contribution, especially regarding internal and customer-related scopes of engineering projects.

\subsubsection{Fundamentals of requirements engineering and systems engineering}

In common engineering and development process models, requirements engineering and its related activities form the initial stage, incorporating a high degree of importance (Eigner, 2014). Following the definition of Dick et al. (2017), RE comprises several activities during product development including requirements discovery, analysis, and qualification. Throughout the development process, requirements are elicited from internal and external sources, which are summarized as stakeholders (Dick et al., 2017; Gilz, 2014). Anyway, elicitation - or in general - gathering requirements is not trivial, which also leads to failure of development projects, caused by insufficient or incorrect RE activities. The process can take a long time, since the granularity of customer demands needs to be defined according to the respective situation (Dick et al., 2017) and may change throughout the process (Prakash and Prakash, 2018). While elicitation and documentation feature one fundamental part of RE, tracing stakeholder demands into system requirements is another, even more demanding task (Dick et al., 2017). Those stakeholder requirements, which are partly available as fully quantified data sets, are transformed into system specifications afterwards. This may be done by simultaneous correlation of requirements with a system model by means of data flow and use-case diagrams (Dick et al., 2017; Gilz, 2014), apart from an option to request admission or rejection from the customer. This is an adequate alternative for an enhanced customer integration bearing trade-offs concerning data integrity (Prakash and Prakash, 2018). Thus, fundamental system considerations become more and more one major aspect and benefit of general model-based systems engineering (MBSE) approaches. MBSE offers a formalized methodology of systems development to eliminate unnecessary (real) components through abstraction. Graphical, mathematical or physical models regarding concept, phenomenon, relationship and structure are applied system-wide, accompanied by several tools for IT-based support.

Specific modelling languages (Eigner, 2014) unburden the development and documentation of progress in all stages from requirements specification until release, especially by vertical integration of system and operational models. The objective in this case is to facilitate understanding, aid in decision-making and explain, control as well as predict occurrences throughout the life cycle. RE is a most significant influence factor in MBSE regarding the initial definition of the system architectural model (addressing function/behavior, performance, structure, and further multiple aspects). In this context, software tools support the RE process by providing model-based use-case definition and traceability links (Dick et al., 2017) on a generalized UML or SysML basis (Gilz, 2014; Dick et al., 2017).

\subsubsection{Requirements engineering for additive manufacturing applications}

Due to the availability of rapid prototyping, quick iterations during product creations become possible, additionally with reduced risk of mainly economic failure. Following the literature, requirements engineering for AM is primarily driven by core and subsidiary-process-oriented implications (Gibson, 2015). Gebhardt (2016) gives a brief overview of aspects, which become necessary with the maturation of AM and the inherent shift in technology-specific demands. Focusing on the technology itself, these aspects can be referred to as guidelines applied within product development and ensuring the consideration of various process related issues. Regarding the applicability of AM for parts and assemblies in general, Schmidt (2016) provides a broad range of relevant requirements, spanning from design and functionality towards economic limitations. Those are necessary for product as well as business success, and therefore vital to both producer and customer. Concerning the necessity to 
master design for AM and the inherent manufacturing processes, Emmelmann et al. (2017) underline the importance of design limitations originating from the different process principles. Process-based constraints provide a basis for requirements specification regarding design, namely features and their geometrical characteristics. Emmelmann et al. (2017) and Kranz (2017) visualize and prove this concern by providing design catalogues for AM parts and assemblies. Thereby, Kranz (2017) focuses on generating material- and process-oriented design guidelines simultaneously. This further clarifies the crucial difference between additive and conventional manufacturing routes. Conventional processes are well established and manufacturers possess notable process knowledge. In contrast, AM imposes great challenges to a successful combination of design and manufacturability that depends on a variety of parameters. Opportunities offered by conventional technologies are constrained by means of minimum radii, minimum allowable wall thickness, undercuts and other geometric features. Often, but not in any given case, additive processes provide even more extensive potentials, which must be assessed and exploited. Therefore, traditional manufacturing cannot equal AM, neither in terms of product requirements, nor regarding process demands. In general, much of the rather scientific literature focuses on the description of processual and design-related requirements without really pursuing distinct fields of requirements elicitation and specification. However, requirements engineering with regard to early process chain and life cycle consideration is of vital importance to the success of an AM related development project. Mies et al. (2016) underline this demand in the context of AM informatics, stressing the necessity to capture and store information on feasible part geometry, suitable materials, AM core processes as well as post-processing. Proceeding from purely technical and technological requirements acquisition towards soft requirements specification, customer participation in design becomes of interest in the AM context. Morar and Kemper (2016) emphasize the promoting character of AM in the context of customer integration. This helps to avoid misunderstandings and major design flaws in early stages, and thus facilitates a quickening of development and fosters short iterations and minor change cycles.

\subsection{Current deficiencies regarding requirements in the context of AM}

Additive manufacturing combines an influential design driver with a direct manufacturing technology, imposing new challenges on both developers and development processes. A significant proportion of research contributions therefore focuses on design and application aspects in the AM environment (Morar and Kemper, 2016; Emmelmann et al., 2017; Kranz, 2017), as well as mastering the respective coreprocesses. However, aspects like process chain considerations and organizational necessities appear to be represented insufficiently. Due to the principles of AM and the necessity to manage part design and process layout simultaneously, those fields must be considered simultaneously. Moving on along the process chain, validation and qualification outline a critical stage in AM product development. So far, those aspects are treated on a more or less generalized level, without differentiation between business branches (Deloitte, 2017). This bears deficiencies in terms of process chain layout and strategic determination of efforts, and decreases profitability - a challenge the AM industry still struggles to meet. In cooperation with industrial partners, the authors have observed that the above-mentioned issues can be identified in lacking knowledge on the users' side, which complicates communication of both user and researcher. According to the aforementioned findings, and due to the fact that AM cannot be reduced to either the design or the manufacturing side, the authors stress an overarching assessment of AM in its context. This contribution therefore presents a multilateral requirements specification approach, in which strategic and organizational aspects are considered alongside typical design and core-process-related demands. Requirements on the organizational level are interpreted herein as, for example, legislative and service-related content, whereas strategic requirements will focus on the application of specific AM technologies in distinct fields of operation with regard to anticipated future deployments. In doing so, the authors foster a target-oriented requirements engineering process, leading towards a more accurate assessment of the overall situation. This facilitates a more realistic estimation of required efforts during the development process.

\section{PROCESS-CHAIN-ORIENTED REQUIREMENTS ENGINEERING FOR AM}

The following section gives an overview of a process-chain oriented requirements engineering approach for AM technologies and applications. At first, a widespread view on the topic enables an overarching assessment of demands and possibilities, as to highlight a precise procedure to identify technological and technical demands thereafter. 


\subsection{Specification throughout the development process}

According to the previously determined difficulties and peculiarities of AM and its environment, the authors propose an approach that is oriented at a systems engineering (SE) level. Following the ISO/IEC/IEEE (2015), the different process types are agreement, organizational project-enabling, technical management and technical operations. The herein presented requirements engineering approach correspondingly adopts this differentiation. It splits the complete spectrum into organizational aspects, functional elements and structures, as well as geometric and processual specifications. Consequently, not only the frequently treated geometric domain is in focus, but also parallel, organizational and management-characterized processes. Those reveal significant influence on project planning and implementation in the additive industrial environment. Figure 1 shows a sequentially depicted overview of the framework.

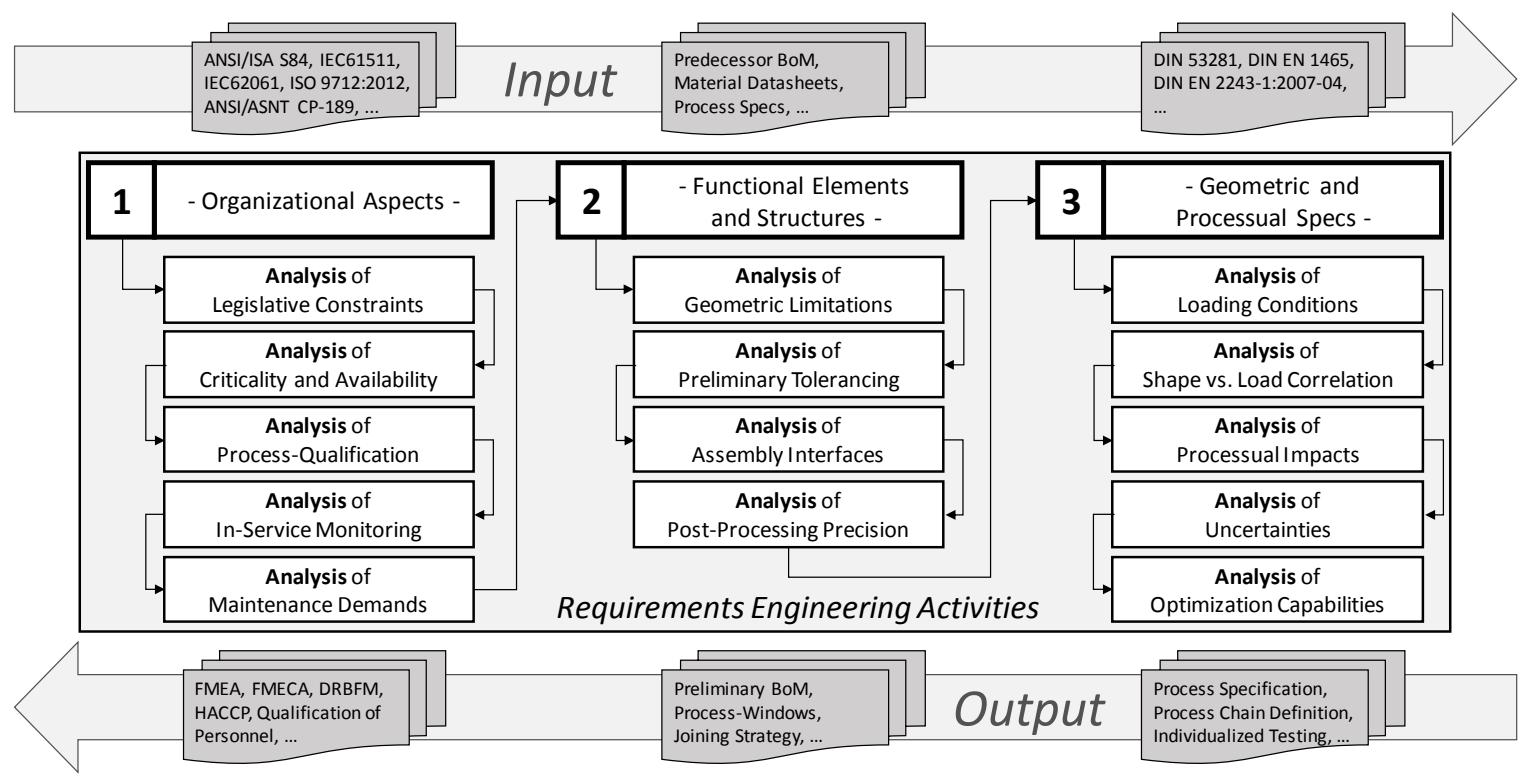

Figure 1. Scope of requirements specification (sequential illustration)

Considering aspects such as legislative constraints (e.g., international law and homologation of passenger vehicles following UN ECE regulations), the criticality and maintainability of parts and systems, as well as in-service monitoring of products is highly necessary for several reasons. First, developers are able to ensure that development targets match with the required preconditions and levels of technological skills. These are essential for an adequate and thorough preparation of process chains, workflow, and personnel according to the demands raised from the customer side. This may also include official regulations, such as those concerning personnel qualification and certification standards (e.g., ANSI/ASNT CP-189, ANSI/ASNT ILI-PQ-2010, and ISO 9712:2012). Second, impacts that might occur during the use-phase of the product can be assessed early and very accurately by the use of reference databases and international standards. Secondary effects showed up in the early phase of product development, allowing for the determination of appropriate prophylaxis or suitable countermeasures. Standards and guidelines that might be of interest in this context may contain further information on functional safety, failure mode and effect analyses (FMEA), and safety integrity requirements. The outcomes of the first stage may include a holistic System-FMEA, design reviews, and a detailed list of critical points and areas of particular testing efforts.

The second stage comprises functional elements and structures within a product or system. Here, geometric requirements on part and assembly level, preliminary toleration and assembly interfaces are of special interest. Coherently, this stage covers demands on post-processing precision, which is essential, considering the intended location and function of the part. Helpful input data of the second stage are manifold, for example, predecessor bills of material $(\mathrm{BoM})$, common material datasheets, and process specifications. Those especially require a high degree of knowledge in a company's own process portfolio, including manufacturing lines and specific tools. As outcomes, preliminary bills of material, preliminary process parameter ranges, as well as joining strategies can be determined. Following this merely functional viewpoint, the third stage focuses on geometry and processual requirements and peculiarities in depth. Typical aspects like loading conditions, topology optimization capabilities and 
shape-correlated loading capacity need to be determined and considered accurately. Here, the developer always needs to assess personnel capabilities (expertise) in order to create a well-established design process without being likely to fail because of missing or insufficient knowledge in structural FEA or soft-kill-option optimization, for example. Moreover, processual specifications need to be assessed according to a much more sophisticated granularity in this stage. Typical requirements regarding processual interfaces relate to part positioning and operation principle in the respective process, as well as accessibility with robotic manipulators (e.g., jaw chucks or end effectors on 3D-robots). Furthermore, an analysis and assessment of uncertainties completes the stage. In the herein presented approach, uncertainties are supposed to be universal without limitation to a specific aspect. Another even more relevant aspect for AM process chains, and the authors' research project in particular, is testing and validation of parts and structures after the primary manufacturing process. Concerning validation, there are many approaches that focus on a holistic and thorough testing strategies, including techniques like thermography, ultrasonic or acoustic emission testing as well as micro-CT and magnetic flux leakage testing to specify AM parts' mechanical and material properties accurately. In this context, the authors stress a profound estimation of relevant, required efforts and achievable benefits arising from the implementation of testing and validation techniques. The use of international standards for certain activities can support this process by provision of situation-specific testing requirements and process parameter ranges (e.g., fabrication and geometric features of test specimen). The outcomes of stage three shall include precise process chain definitions and process step specifications, as well as individual testing and validation approaches. Moving on further, the resulting requirements of each stage are supposed to be fed back in CAD part data of multiple kinds, machine databases as well as working instructions provided to workers, for example. Feeding back data in information systems provides high compatibility with SE approaches in enterprises. Requirements elicitation and specification are supported by an IT environment, which can be directly linked with established information systems in companies. The sequential model as depicted in Figure 1 must not be passed strictly sequential but may be adapted to the respectively established development process.

\subsection{The requirements-funnel and its application}

To facilitate handling and application of the presented requirements engineering approach, the authors include a prioritization scheme, which enables target-oriented specification. The main goal of this procedure is to generate knowledge of requirements by gradual refinement of boundary conditions and successive narrowing of the developers and customers viewpoint. Figure 2 gives a comprising overview of the procedure depicting it as a "funnel", due to the channeling and narrowing character of the process. It is supposed to be conducted in very close collaboration between the development team and the respective customer. This is not limited to only one certain field of interest (e.g., mechanical design), but also covers the aforementioned aspects in their entirety. It might occur that the customer is, due to its individual degree of foreknowledge, overcautious and prejudiced against the implementation of a new - and in the case of AM rather unconventional - technology. This bears impediments, which are amplified by an insufficient overview of the technological situation, especially regarding feasibility of design concepts and the real capability of AM processes. Together with a high level of risk awareness in today's competitive economic environment, reservations against AM as a performance enabler arise and prevail. The authors have identified this coherence as most influential in different collaborations with industrial partners. Besides the funnel, Figure 2 shows the surroundings that contribute to the process. Generally, the infrastructure is a server- or cloud-based MBSE and PLM-environment, incorporating databases of different kinds.

First, legal premises and demands (administrations act as stakeholders in any type of product) provide a basis for product design by means of actual access to markets. Observance of legislation is stringently required, wherefore infringements concerning legal demands lead to a reset of development activities without exception. A second escalation is given by international standards and proprietary norms (e.g., DIN, ISO or ASTM). In addition, individual and generalized process portfolio analyses are mirrored in the IT backbone. This provides a maximum of diversity and reliability in terms of process selection and production planning, including established solutions, a wide variety of AM technologies and machines, as well as available testing technologies. Here, specifically material- and geometry-related requirements and limitations are taken into consideration in order to align design, processing and validation of AM parts, which is highly required as emphasized before. The requirements-funnel initiates the clarification and specification process by settling the industrial branch considered. The following steps contribute to 
the above-mentioned gradual refinement of requirements, going along with an incremental reduction of remaining entrepreneurial risks. By the definition of intended lot sizes and safety demands, process selection is supported in a first instance. The definition of loading conditions, and inherently material requirements, narrows suitable processes and process chain elements further to be integrated in the aspired enterprises workflow. Here, the aforementioned consideration of correlations between processing, post-processing and testing activities yields the strongest influence. Definition of design spaces to be considered and a rough initial process chain round out the specification process. The herein presented procedure does not raise a claim towards completeness, as it is an ongoing process and the authors will extend it to a higher degree with proceeding research efforts in the field.

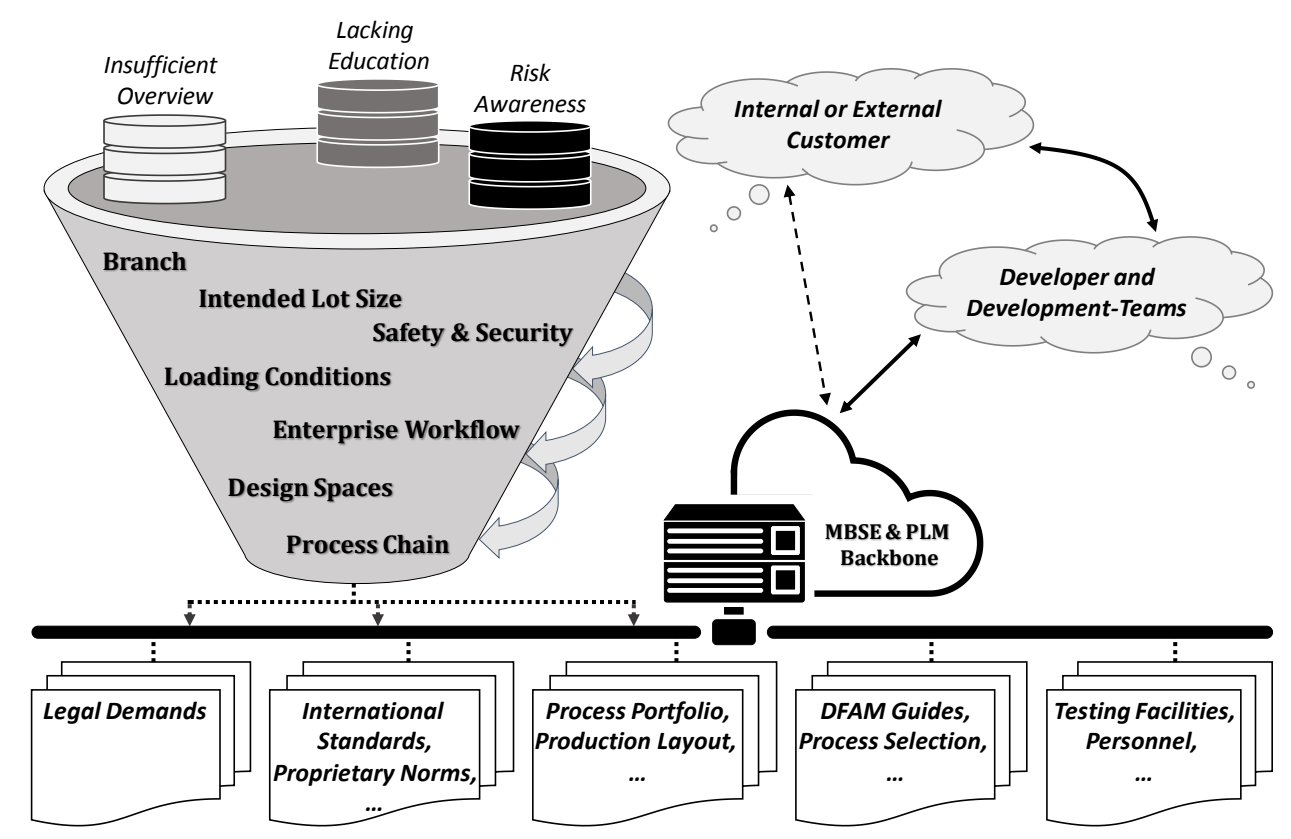

Figure 2. Requirements-funnel for developer-oriented/driven requirements specification

\section{APPLICATION EXAMPLE}

To validate the framework with its opportunities and advantages, the authors give an illustrative example from the automotive sector. There are different approaches towards design and manufacture of the body in white (BIW), featuring powder-bed-based processes like SLM for specific use-cases. However, those procedures require high efforts, and impose strict constraints at the same time, especially regarding flexibility and build spaces of the process. The herein analyzed section constitutes a connecting node (joint section design) between the lower and upper load path in a passenger vehicle, including one of the lower crossbeam members, considering variant-specific local reinforcements and stiffening.

Following the framework shown in Figure 1, organizational aspects are analyzed in the first instance. Regarding legislative constraints, current laws act as the baseline requirements, including crash demands as well as passenger and pedestrian safety issues. Since the connecting node is one part of the major structure of the BIW, criticality and availability is high (i.e. accessibility of spare parts in case of replacement). Due to the plant layout, internal high pressure forming for tubular bodies as well as casting processes are available; however, the company has not established AM by now. Since there is only a small series of cars and for promotion of knowledge acquisition, quality assurance and in-service monitoring shall be possible with reasonable effort, while there shall be no need for interval replacement of the part. Considering functional elements and structures, geometric limitations lie within the design space size, which is, for example, around $250 \mathrm{~mm} \times 200 \mathrm{~mm} \times 200 \mathrm{~mm}$. In order to connect to surrounding structural members, tolerances must be rather tight, especially concerning form deviations. This applies to the non-trivial, poka-yoke assembly interfaces, which ascertain minimum failure-rate in assembly. Regarding geometric and processual aspects, loading conditions prevail from the general vehicle specification. Due to the low level of process knowledge, uncertainties and processual impacts cannot be foreseen by now, wherefore a robust process chain design is strongly required.

There are different solutions to the task, as depicted in Figure 3, such as a hybrid-manufactured node [1], relying on semi-finished parts and downstream LMD build-up, or an SLM fabricated node [2] with a 
bionics-inspired shape and internal lattice structures, or. On the left, there are the major load path, as well as cross beam members, divided by the primary build space (dark grey portion). The middle section shows the connecting node as an LMD-hybrid [1] (dark shade shows the semi-finished part, white portions are fabricated using LMD), whereas on the right the SLM manufactured node [2] is depicted.
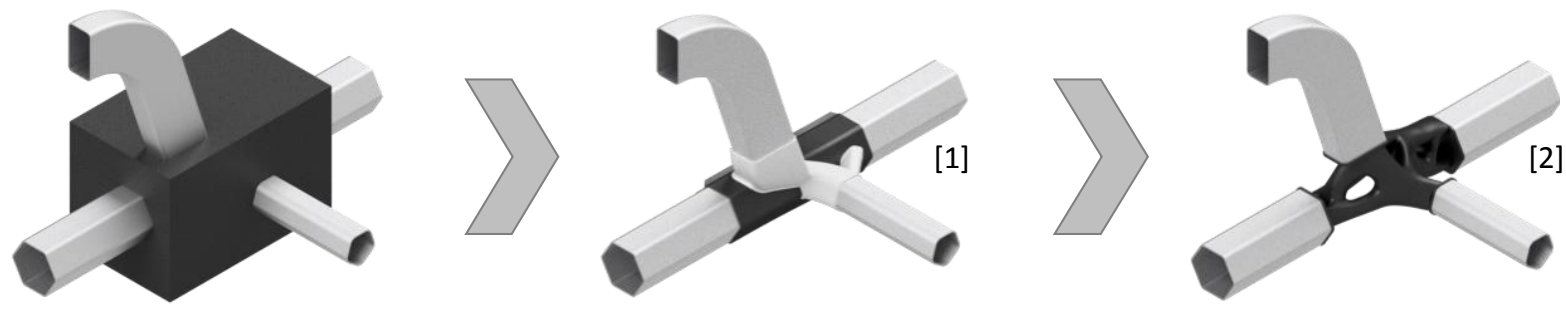

Figure 3. Schematic realization of a connecting node in the front-end section of the BIW

For the specification profile presented above, both principles would be suitable in terms of technical value and satisfaction of geometric requirements. However, looking at the capability to produce accurate, easy to manufacture and less testing-intense parts, hybrid processes permit potentially better alternatives. First, semi-finished products out of internal high pressure forming processes provide rather tight tolerances and well-defined material characteristics at reasonable economic conditions. The conventional process step can realize large portions of the part, reducing efforts in downstream hybrid manufacturing significantly. Second, diverse manufacturing strategies ascertain geometric flexibility due to individual adaptability of parts (e.g., regarding end effectors on 3D robots). Regarding inspection issues, simple geometries allow for better handling and more reliable test results especially in the light of new manufacturing routes. The example shows the potential of thorough requirements engineering in AM environments, allowing for more distinct and need-based assessment of additive processes and process chain solutions. Further examinations and examples by the authors will treat the complex correlations within hybrid manufacturing processes, especially focusing individual process assessment and selection, as well as interface design and its secondary effects on downstream activities.

\section{DISCUSSION AND OUTLOOK}

In this contribution, the authors present a requirements specification framework that aims to involve developers and customers in the development process of additively manufactured products and systems. Owing to the manifold opportunities in the AM environment, the procedure offers a generic way to assess solutions for design and process planning issues. Herein, multilateral process chain impacts from the manufacturing and post-processing position are considered along with an initial analysis of testing-induced requirements. The framework refers to established systems engineering approaches by means of logical coherences and data structures. According to the target of systems engineering, to provide continuous model and data flows during the development process, the authors stressed a server- or cloud-based IT infrastructure by means of databases and decision support systems. By gradually refining customer demands, the process is relevantly supported with the help of legislative boundaries, international standards and a thorough analysis of existing process portfolio. However, the proposed framework is an ongoing process, which is not intended to be complete and finished yet. A more widespread consideration of mutual influences throughout development, manufacturing and use-phase still needs to be implemented in the consecutive steps of the framework. Coherently, and as to ascertain testability and compatibility, the authors stress an enhanced process and material match determination, which they have already introduced previously (Kaspar et al., 2018).

Regarding testing and validation, particular attention lies on a generalized definition of correlations between design, material, process layout and testability. In doing so, the authors aim to provide a method to weigh testing efforts and expenses against advantages that can be achieved. Moreover, the fundamental data supply and storage for the presented framework must be elaborated in terms of primary scope, verbosity, complexity and accessibility for the users.

\section{REFERENCES}

Brandt, M. (2017), “The role of lasers in additive manufacturing”, In: M. Brandt, (Ed.), Laser Additive Manufacturing: Materials, Design, Technologies, and Applications, Woodhead Publishing, Duxford, pp. 1-18. https://doi.org/10.1016/C2014-0-03891-9. 
Deloitte (2017), 3D opportunity for standards - Additive manufacturing measures up, Deloitte Consulting LLP, Deloitte Insights.

Dick, J., Hull, E. and Jackson, K. (2017), Requirements Engineering, Springer International Publishing, Cham. https://doi.org/10.1007/978-3-319-61073-3.

Eigner, M. (2014), “Überblick Disziplin-spezifische und -übergreifende Vorgehensmodelle”, In: M. Eigner, D. Roubanov and R. Zafirov, (Ed.), Modellbasierte Virtuelle Produktentstehung, Springer-Verlag, Berlin Heidelberg, pp. 53-75. https://doi.org/10.1007/978-3-662-43816-9.

Emmelmann, C., Herzog, D. and Kranz, J. (2017), "Design for laser additive manufacturing”, In: M. Brandt, (Ed.), Laser Additive Manufacturing: Materials, Design, Technologies, and Applications, Woodhead Publishing, Duxford, pp. 259-279. https://doi.org/10.1016/C2014-0-03891-9.

Gebhardt, A. (2016), Additive Fertigungsverfahren, Carl Hanser Verlag, München. https://doi.org/10.3139/9783446445390.

Gilz, T. (2014), "Requirements Engineering und Requirements Management”, In: M. Eigner, D. Roubanov and R. Zafirov, (Ed.), Modellbasierte Virtuelle Produktentstehung, Springer-Verlag, Berlin Heidelberg, pp. 53-75. https://doi.org/10.1007/978-3-662-43816-9.

Graf, B., Marko, A., Petrat, T., Gummenyuk, A. and Rethmeier, M. (2018), “3D laser metal deposition: process steps for additive manufacturing”, Welding in the World, Vol. 62, pp. 877-883. https://doi.org/10.1007/s40194-018-0590-x.

Gu, D. (2015), Laser Additive Manufacturing of High-Performance Materials, Springer-Verlag, Berlin Heidelberg. https://doi.org/10.1007/978-3-662-46089-4.

Herderick, E.D. and Patterson, C. (2016), "Industrial Implementation of Additive Manufacturing", In: A. Bandyopadhyay and S. Bose, (Ed.), Additive Manufacturing, CRC Press, Taylor \& Francis, Boca Raton, pp. 259-275.

ISO/ASTM (2015), Standard Terminology for Additive Manufacturing - General Principles - Terminology, vol. 52900:2015-12.

ISO/IEC/IEEE (2015), Systems and software engineering - System life cycle processes, vol. 15288:2015.

Kaspar, J., Stoffels, P., Schneberger, J.-H. and Vielhaber, M. (2018), "Early phase evaluation of additive manufacturing technologies within an integrated product and production engineering approach", DESIGN 2018 15th International Design Conference, Dubrovnik, 21.-24. May 2018, Design Society, pp. 11851196. https://doi.org/10.21278/idc.2018.0467.

Kranz, J. (2017), Methodik und Richtlinien für die Konstruktion von laseradditiv gefertigten Leichtbaustrukturen, Springer-Verlag GmbH. https://doi.org/10.1007/978-3-662-55339-8.

Lorenz, K.A., Jones, J.B., Wimpenny, D.I. and Jackson, M.R. (2015), “A Review of Hybrid Manufacturing”, Proc. of Solid Freeform Fabrication, pp. 96-108.

Loy, J. and Tatham, P. (2016), "Redesigning Production Systems”, In: S. S. Muthu and M. M. Savalani, (Ed.), Handbook of Sustainability in Additive Manufacturing, Springer Science+Business Media, Singapore, pp. 145-171. http://dx.doi.org/10.1007/978-981-10-0549-7_7.

Mahamood, R.M. (2018), Laser Metal Deposition Process of Metals, Alloys, and Composite Materials, Springer, Cham. https://doi.org/10.1007/978-3-319-64985-6.

Mies, D., Marsden, W. and Warde, S. (2016), "Overview of Additive Manufacturing Informatics: A Digital Thread", Integrating Materials and Manufacturing Innovation, Vol. 5 No. 1), https://doi.org/10.1186/s40192-016-0050-7.

Milewski, J.O. (2017), Additive Manufacturing of Metals, Springer International Publishing, Cham. http://dx.doi.org/10.1007/978-3-319-58205-4.

Morar, D. and Kemper, H.-G. (2016), "Requirements of information systems in product development and production regarding additive manufacturing - a quantitative exploration", 24th European Conference on Information Systems, Istanbul, 12.-15. June 2016, Association for Information Systems.

Prakash, N. and Prakash, D. (2018), Data Warehouse Requirements Engineering, Springer Nature, Singapore. https://doi.org/10.1007/978-981-10-7019-8.

Sames, W.J., List, F.A., Pannala, S., Dehoff, R.R. and Babu, S.S. (2016), “The metallurgy and processing science of metal additive manufacturing”, International Materials Reviews, Vol. 61 No. 5, pp. 315-360. https://doi.org/10.1080/09506608.2015.1116649.

Schmidt, T. (2016), Potentialbewertung generativer Fertigungsverfahren für Leichtbauteile, Springer Vieweg, Berlin Heidelberg. http://dx.doi.org/10.1007/978-3-662-52996-6.

Schmidt, M., Merklein, M., Bourell, D., Dimitrov, D., Hausotte, T., Wegener, K., Overmeyer, L., Vollertsen, F. and Levy, G. (2017), "Laser based additive manufacturing in industry and academia", Cirp Annals, Vol. 66 No. 2, pp. 561-583. https://doi.org/10.1016/j.cirp.2017.05.011.

\section{ACKNOWLEDGMENTS}

The authors thank the European Regional Development Fund (ERDF) for supporting their AM research along with the enhanced cooperation between both universities within the project 14.2.1.4 - 2017/3. 